\title{
Feasibility and Effectiveness of an Automated Bilingual Text Message Intervention for Weight Loss: Pilot Study
}

Julia K Kolodziejczyk ${ }^{1,2,3}$, MS; Gregory J Norman ${ }^{1,2}, \mathrm{PhD}$; Angelica Barrera-Ng ${ }^{1,2}$; Lindsay Dillon ${ }^{1,2}$, MPH; Simon Marshall ${ }^{1,2}$, PhD; Elva Arredondo ${ }^{3}, \mathrm{PhD}$; Cheryl L Rock ${ }^{2}, \mathrm{PhD}, \mathrm{RD}$; Fred Raab ${ }^{1,2}$, AAS; William G Griswold ${ }^{4}$, PhD; Mark Sullivan $^{1,2}$, MS; Kevin Patrick ${ }^{1,2}$, MD, MS

${ }^{1}$ Center for Wireless \& Population Health Systems (CWPHS), Qualcomm Institute/Calit2, University of California, San Diego, La Jolla, CA, United States

${ }^{2}$ Department of Family \& Preventive Medicine, University of California, San Diego, La Jolla, CA, United States

${ }^{3}$ Graduate School of Public Health, San Diego State University, San Diego, CA, United States

${ }^{4}$ Computer Science \& Engineering, University of California, San Diego, La Jolla, CA, United States

\section{Corresponding Author:}

Julia K Kolodziejczyk, MS

Center for Wireless \& Population Health Systems (CWPHS), Qualcomm Institute/Calit2

University of California, San Diego

9500 Gilman Drive, Dept 0811

La Jolla, CA, 92093

United States

Phone: 18585349550

Fax: 18585349404

Email: jkolodzi@ucsd.edu

\section{Abstract}

Background: Little is known about the feasibility and acceptability of tailored text message based weight loss programs for English and Spanish-language speakers.

Objective: This pilot study evaluated the feasibility, acceptability, and estimated impact of a tailored text message based weight loss program for English and Spanish-language speakers. The purpose of this pilot study was to inform the development of a full-scale randomized trial.

Methods: There were 20 overweight or obese participants (mean age 40.10, SD 8.05; 8/20, 40\% male; 9/20, 45\% Spanish-speakers) that were recruited in San Diego, California, from March to May 2011 and evaluated in a one-group pre/post clinical trial. For 8 weeks, participants received and responded to 3-5 text messages daily sent from a fully automated text messaging system. They also received printed weight loss materials and brief 10-15 minute weekly counseling calls. To estimate the impact of the program, the primary outcome was weight $(\mathrm{kg})$ measured during face-to-face measurement visits by trained research staff. Pre and post differences in weight were analyzed with a one-way repeated measures analysis of variance. Differences by language preference at both time points were analyzed with $t$ tests. Body mass index and weight management behaviors also were examined. Feasibility and acceptability were determined by recruitment success, adherence (ie, percentage of replies to interactive text messages and attrition), and participant satisfaction.

Results: Participants who completed the final assessment $(\mathrm{N}=18)$ decreased body weight by $1.85 \mathrm{~kg}\left(F_{1,17}=10.80, P=.004, \mathrm{CI}_{\Delta}\right.$ 0.66-3.03, $\left.\eta^{2}=0.39\right)$. At both time points, there were no differences in weight by language preference. Participants responded to $88.04 \%$ (986/1120) of interactive text messages, attrition rate was 10\% (2/20), and 94\% (19/20) of participants reported satisfaction with the program.

Conclusions: This fully automated text message based weight program was feasible with English and Spanish-speakers and may have promoted modest weight loss over an 8-week period.

Trial Registration: Clinicaltrials.gov NCT01171586; http://clinicaltrials.gov/ct2/show/NCT01171586 (Archived by WebCite at http://www.webcitation.org/6Ksr6dl7n).

(JMIR Res Protoc 2013;2(2):e48) doi: 10.2196/resprot.2789 


\section{KEYWORDS}

physical activity; diet; obesity; health behavior; Hispanic Americans; weight loss; cellular phone; text messaging

\section{Introduction}

Text message based programs to promote behavior change are a rapidly growing area of research. This inexpensive, instantaneous, two-way communication of brief written messages via a mobile phone has many capabilities that may be useful for promoting weight loss. For example, texting features can support important constructs in behavior change theories such as cues to action, reinforcement, goal setting, goal reminders, and feedback. Text messages can be used as a stand-alone program [1-4] or can be integrated easily with other wireless or networked technologies [5,6]. Studies have demonstrated that text messages promote improved diet [5], increased physical activity (PA) [4,7-10], behavioral strategies like self-monitoring [3], and weight loss [1,2,6,11,12]. However, more research is needed regarding long-term efficacy and best practices of these programs, in particular in diverse populations.

This pilot study evaluated feasibility and acceptability of a tailored text message based weight loss program for English and Spanish-language speakers and enabled an estimate as to its impact on weight status. The purpose of this pilot study was to inform the development of a full-scale randomized trial.

\section{Methods}

\section{Unblinded One-Group Pre/Post Design}

We used an unblinded one-group pre/post design. The Institutional Review Board of University of California, San Diego (UCSD) approved this study. This manuscript is in accordance with the CONSORT-EHEALTH checklist [13] and is a registered trial (NCT01171586).

\section{Recruitment}

Participants were recruited in San Diego, California, from March to May 2011 via newspapers, flyers, online announcements, and participant recommendations. The first 20 individuals who met the eligibility criteria were enrolled, with a goal of $40 \%$ $(8 / 20)$ male and 50\% (10/20) self-identified Spanish-speaking. Bilingual speakers choose language-message preference.

Eligible individuals were 21-60 years of age, had a body mass index (BMI) of 27.0-39.9, had a cellphone capable of sending and receiving text messages, were current users of texting or willing/able to learn, and could communicate in English or Spanish. Participants were excluded if they could not engage in moderate intensity PA, were pregnant or intended to become pregnant during the study, had a history of substance abuse or psychiatric disorders that would impair compliance, were using weight-altering medications, or were enrolled in another weight loss program. At baseline, potential participants were screened for inclusion and exclusion criteria and underwent written informed consent. Participants were compensated \$75 for participation and $\$ 10$ for a text message plan.

\section{Intervention}

Social cognitive theory [14], control theory [15], and ecological theory [16] informed the intervention. It integrated these theoretical approaches with evidence-based behavioral strategies for improving diet and PA. Strategies include self-monitoring, intention formation, goal setting, goal review, feedback on performance, self-efficacy, benefits, barriers, problem-solving, social support, and tailoring.

The 8-week intervention included: (1) 3-5 automatically scheduled and tailored text messages per day. Message content focused on diet and PA weight management behaviors and strategies; (2) a printed weight loss binder organized by weekly weight management topics such as portion control, increasing PA, reducing sedentary behavior, and self-monitoring; and (3) brief weekly 10-15 minute counseling calls to provide encouragement and reinforcement. A database was developed of more than 3000 text messages. The research group translated and culturally tailored the messages to Spanish-speakers to ensure linguistic and cultural equivalence. Approximately one-quarter of messages requested a reply, with the balance providing tips, suggestions, and positive reinforcement or encouragement for improved behaviors. The following shows sample messages sent and received from ConTxt (San Diego, CA 2011): (1) ConTxt-What is your weight today?, (2) Participant-220, (3) ConTxt-Congratulations! You have lost 5 lbs since starting ConTxt, (4) ConTxt-Here's a healthy tip, put your pedometer on your nightstand so you can remember to put it on in the morning, (5) ConTxt-Work on your goal of reducing portion sizes this week by buying single serving pre-packaged snacks, and (6) ConTxt-Thank you. Your response has been recorded. A total of 1500 rules were added to control what message was sent based on the weekly behavioral strategy, day of the week, and time of day, as well as other parameters such as self-reported weight management behaviors and pedometer step count. A baseline dietary assessment of weight management behaviors was conducted using the Weight Behavior Inventory (WBI) to identify unique diet and PA behavior challenges for each participant contributing to high-energy intake and low-energy expenditure. A computerized expert system processed these data to create individualized goals based on predetermined logic rules. Goals were presented to the user via text message to serve as prompts for behavioral improvements. The system is designed so participants who show rapid and sustained progress can advance through content, while those experiencing difficulties can receive additional tips and suggestions.

\section{Measures}

Outcomes were measured at baseline and 8 weeks by trained research staff at UCSD research offices during face-to-face visits. The primary outcome was weight $(\mathrm{kg})$ measured using a calibrated scale. Secondary measures included BMI calculated as $\mathrm{kg} / \mathrm{m}^{2}$ and weight management behaviors associated with weight loss measured with the 35-item WBI (validation study under review, Kolodziejczyk et al 2013). The WBI was adapted 
from the validated Eating Behavior Inventory [17,18]. Each behavior on the WBI is rated on a five-point scale. Total scores are averaged and can range from 1-5. Sample items include "I keep one or two raw vegetables available for snacks" and "I decide ahead of time what I will eat for meals and snacks."

Feasibility and acceptability were measured by recruitment success, adherence, and participant satisfaction. Recruitment was deemed successful if we achieved our enrollment goal of 20 participants in two months. Adherence was measured by percentage of replies to interactive text messages (ie, text messages requesting a reply) and attrition rate. Satisfaction was measured using a Likert scale that asked about level of satisfaction with the program, as well as program components. In addition, we asked open-ended questions about elements of the program such as what they liked the least and best.

\section{Statistical Analysis}

Pre and post differences between weight, BMI, and WBI scores were analyzed with one-way repeated measures analysis of variance. WBI score differences between gender and language preference at each time point were analyzed with independent sample $t$ tests. Analyses used an alpha level <.05 and were conducted using SPSS Statistics 17.0 (SPSS Inc, Chicago, Illinois).

\section{Results}

\section{Participants}

A total of 18 out of the 20 participants completed all measures (ie, two participants completed the program but did not show for their final assessment). On average, the sample was obese and had approximately equal percentages of participants across demographic categories. Table 1 displays participant demographics.

Table 1. Demographic characteristics of the ConTxt pilot participants $(\mathrm{N}=20)$.

\begin{tabular}{|c|c|c|}
\hline Demographic variables & & Overall sample \\
\hline Age at study entry in years, mean (SD) & & $40.10(8.05)$ \\
\hline BMI $\left(\mathrm{kg} / \mathrm{m}^{2}\right)$, mean $(\mathrm{SD})$ & & $33.67(4.00)$ \\
\hline Female, n $(\%)$ & & $12(60)$ \\
\hline \multicolumn{3}{|l|}{ Education, $\mathbf{n}(\%)$} \\
\hline & Trade or technical school & $6(30)$ \\
\hline & Some college & $2(10)$ \\
\hline & College graduate & $3(15)$ \\
\hline & Graduate degree & $7(35)$ \\
\hline & "Prefer not to answer" & $2(10)$ \\
\hline Married, n (\%) & & $11(55)$ \\
\hline \multicolumn{3}{|l|}{ Race/ethnicity, $\mathbf{n}(\%)^{\text {a }}$} \\
\hline & Hispanic & $15(75)$ \\
\hline & White non-Hispanic & $13(65)$ \\
\hline & Asian & $2(10)$ \\
\hline & "Prefer not to answer" & $4(20)$ \\
\hline \multicolumn{3}{|l|}{ Language preference, $\mathrm{n}(\%)$} \\
\hline & English & $11(55)$ \\
\hline & Spanish & $9(45)$ \\
\hline \multicolumn{3}{|l|}{ Monthly income, n (\%) } \\
\hline & $\$ 1,000-1,999$ & $5(25)$ \\
\hline & $\$ 2,000-3,999$ & $5(25)$ \\
\hline & $\$ 4,000-5,999$ & $6(30)$ \\
\hline & $\geq \$ 6,000$ & $3(15)$ \\
\hline "Don't know/prefer not to answer" & & $1(5)$ \\
\hline
\end{tabular}

${ }^{\mathrm{a}}$ More than one race category may apply 


\section{Participants' Body Weight}

Participants decreased body weight by $1.85 \mathrm{~kg}\left(F_{1,17}=10.80\right.$, $\left.P=.004, \mathrm{CI}_{\Delta} 0.66-3.03, \eta^{2}=0.39\right)$, decreased BMI by $0.70 \mathrm{~kg} / \mathrm{m}^{2}$ $\left(F_{1,17}=13.21, P=.002, \mathrm{CI}_{\Delta} 0.29-1.11, \eta^{2}=0.44\right)$, and increased WBI scores by 0.56 points $\left(F_{1,17}=14.51, P=.001, \mathrm{CI}_{\Delta} 0.25-0.87\right.$, $\eta^{2}=0.46$ ) (Table 2). At baseline, there were no differences in WBI scores by gender $\left(t_{18}=0.71, P=.48, \mathrm{CI}_{\Delta}-0.31\right.$ to 0.62$)$. There were no baseline differences by language preference and weight $\left(t_{18}=0.14, P=.89, \mathrm{CI}_{\Delta}-15.44\right.$ to 17.60$)$, $\mathrm{BMI}\left(t_{18}=-0.51\right.$,
$P=.61, \mathrm{CI}_{\Delta}-4.80$ to 2.91$)$, or WBI scores $\left(t_{18}=1.01, P=.33, \mathrm{CI}_{\Delta}\right.$ -0.23 to 0.67$)$. At 8 weeks, there were no differences in WBI scores by gender $\left(t_{16}=0.81, P=.43, \mathrm{CI}_{\Delta}-0.54\right.$ to 0.24$)$, but participants preferring Spanish language had higher WBI scores (mean 2.87, SD 0.33) than English-preference participants (mean 2.48 , SD $0.32 ; t_{16}=-2.60, P=.02, \mathrm{CI}_{\Delta}-0.72$ to -0.07$)$. There were no differences at 8 weeks by language preference and weight $\left(t_{16}=0.07, P=.95, \mathrm{CI}_{\Delta}-19.16\right.$ to 20.47$)$ or $\mathrm{BMI}$ $\left(t_{16}=-0.36, P=.73, \mathrm{CI}_{\Delta}-5.34\right.$ to 3.80$)$.

Table 2. Weight, BMI, and WBI from the ConTxt pilot study (San Diego, CA 2011) at baseline and 8 weeks (N=18).

\begin{tabular}{llll}
\hline Outcome & Baseline & 8 weeks & $\%$ Change \\
\hline Weight $(\mathrm{kg})^{\mathrm{a}}$, mean $(\mathrm{SD})$ & $92.96(39.65)$ & $91.11(42.41)^{\mathrm{b}}$ & -1.99 \\
BMI $\left(\mathrm{kg} / \mathrm{m}^{2}\right)$, mean (SD) & $33.78(4.16)$ & $33.07(4.45)^{\mathrm{b}}$ & -2.10 \\
WBI score (points) & $2.11(0.49)$ & $2.67(0.37)^{\mathrm{c}}$ & 26.54 \\
\hline
\end{tabular}

${ }^{\mathrm{a}} \mathrm{kg}=$ kilograms

${ }^{\mathrm{b}} P<.01$

${ }^{\mathrm{c}} P<.001$

\section{Participant Interest}

There was considerable interest in the study, as the recruitment goal of enrolling 20 participants in two months was achieved quickly after receiving 123 inquiries. Participants responded to $88.04 \%(986 / 1120)$ of interactive text messages, and there was a low $10 \%(2 / 20)$ attrition rate. Most participants (94\%, 19/20) reported satisfaction with the program. Participants also reported the program helped motivate and reinforce healthier habits and choices $(n=5)$, encouraged portion control and awareness of energy intake $(n=4)$, and taught how to be more active $(n=5)$. Some challenges participants reported included feelings of withdrawal after the program ended $(n=7)$ and technical issues with their phone, which sometimes hindered message response $(n=4)$.

\section{Discussion}

\section{The Weight Loss Program}

An 8-week text message based weight loss program was found to be both feasible and acceptable in terms of recruitment interest, participant adherence, and satisfaction. The program may have had positive effects on weight management behaviors and weight outcomes, although this needs to be confirmed in a study with a stronger design. These results are consistent with previous text message based weight loss studies [1,2,11]. Based on information we received from this pilot, some changes to be implemented in the full-scale trial include user-initiated messages (eg, suggestions for restaurant meals, PA), more message personalization (eg, names of social supporters, PA locations), a greater focus on participant message preference (eg, participants will be able to set text message preferences through the use of a like/unlike system), inclusion of "milestone" and "competitive" messages based on weight and pedometer step count (eg, when a participant reaches a certain milestone, such as five pounds lost, he or she will receive a congratulatory message, and the system will compare the participant's weight loss with the groups' weight loss), and improvements to system programming to reduce technical errors (eg, a participant not receiving the correct follow-up message). In addition, based on feedback from the Spanish-language speaking participants, we made some of the Spanish materials clearer (eg, more pictures, simpler language).

\section{Study Limitations}

Study limitations include a small sample, a short time frame, and a one-group pre/post design. Therefore, our findings are suggestive rather than conclusive. Based upon these pilot study findings a full-scale randomized controlled trial currently is underway enrolling 298 participants for a one-year intervention.

\section{Acknowledgments}

The National Cancer Institute (R01CA138730; Clinical Trial Registration: NCT01171586) supported this project. We gratefully acknowledge the support of everyone at the Center for Wireless \& Population Health Systems.

\section{Conflicts of Interest}

Dr Patrick is co-owner of Santech, Inc, which is developing products related to the research described in this paper. Terms of this arrangement have been reviewed and approved by University of California, San Diego in accordance with their respective conflict of interest policies. Dr Norman, Dr Rock, and Mr Raab have received consulting income from Santech, Inc. 


\section{References}

1. Patrick K, Raab F, Adams MA, Dillon L, Zabinski M, Rock CL, et al. A text message-based intervention for weight loss: randomized controlled trial. J Med Internet Res 2009;11(1):e1 [FREE Full text] [doi: 10.2196/jmir.1100] [Medline: 19141433]

2. Haapala I, Barengo NC, Biggs S, Surakka L, Manninen P. Weight loss by mobile phone: a 1-year effectiveness study. Public Health Nutr 2009 Dec;12(12):2382-2391. [doi: 10.1017/S1368980009005230] [Medline: 19323865]

3. Shapiro JR, Bauer S, Hamer RM, Kordy H, Ward D, Bulik CM. Use of text messaging for monitoring sugar-sweetened beverages, physical activity, and screen time in children: a pilot study. J Nutr Educ Behav 2008;40(6):385-391 [FREE Full text] [doi: 10.1016/j.jneb.2007.09.014] [Medline: 18984496$]$

4. Fjeldsoe BS, Miller YD, Marshall AL. MobileMums: a randomized controlled trial of an SMS-based physical activity intervention. Ann Behav Med 2010 May;39(2):101-111. [doi: 10.1007/s12160-010-9170-z] [Medline: 20174902]

5. Soureti A, Murray P, Cobain M, Chinapaw M, van Mechelen W, Hurling R. Exploratory study of web-based planning and mobile text reminders in an overweight population. J Med Internet Res 2011;13(4):e118 [FREE Full text] [doi: 10.2196/jmir.1773] [Medline: 22182483]

6. Hurling R, Catt M, Boni MD, Fairley BW, Hurst T, Murray P, et al. Using internet and mobile phone technology to deliver an automated physical activity program: randomized controlled trial. J Med Internet Res 2007;9(2):e7 [FREE Full text] [doi: 10.2196/jmir.9.2.e7] [Medline: 17478409]

7. Shapiro JR, Koro T, Doran N, Thompson S, Sallis JF, Calfas K, et al. Text4Diet: a randomized controlled study using text messaging for weight loss behaviors. Prev Med 2012 Nov;55(5):412-417. [doi: 10.1016/j.ypmed.2012.08.011] [Medline: $\underline{22944150]}$

8. Prestwich A, Perugini M, Hurling R. Can implementation intentions and text messages promote brisk walking? A randomized trial. Health Psychol 2010 Jan;29(1):40-49. [doi: 10.1037/a0016993] [Medline: 20063934]

9. Sirriyeh R, Lawton R, Ward J. Physical activity and adolescents: an exploratory randomized controlled trial investigating the influence of affective and instrumental text messages. Br J Health Psychol 2010 Nov; 15(Pt 4):825-840. [doi: 10.1348/135910710X486889] [Medline: 20156396]

10. Fukuoka Y, Vittinghoff E, Jong SS, Haskell W. Innovation to motivation--pilot study of a mobile phone intervention to increase physical activity among sedentary women. Prev Med 2010;51(3-4):287-289 [FREE Full text] [doi: 10.1016/j.ypmed.2010.06.006] [Medline: 20600263]

11. Joo NS, Kim BT. Mobile phone short message service messaging for behaviour modification in a community-based weight control programme in Korea. J Telemed Telecare 2007;13(8):416-420. [doi: 10.1258/135763307783064331] [Medline: 18078554]

12. Park MJ, Kim HS, Kim KS. Cellular phone and Internet-based individual intervention on blood pressure and obesity in obese patients with hypertension. Int J Med Inform 2009 Oct;78(10):704-710. [doi: 10.1016/j.ijmedinf.2009.06.004] [Medline: 19643661$]$

13. Eysenbach G, CONSORT-EHEALTH Group. CONSORT-EHEALTH: Improving and standardizing evaluation reports of Web-based and mobile health interventions. J Med Internet Res 2011;13(4):e126 [FREE Full text] [doi: 10.2196/jmir.1923] [Medline: 22209829]

14. Bandura A. Social foundations of thought and action: a social cognitive theory. Englewood Cliffs, N.J: Prentice-Hall; 1986.

15. Carver CS, Scheier MF. Control theory: a useful conceptual framework for personality-social, clinical, and health psychology. Psychol Bull 1982 Jul;92(1):111-135. [Medline: 7134324]

16. Stokols D. Translating social ecological theory into guidelines for community health promotion. Am J Health Promot 1996;10(4):282-298. [Medline: 10159709]

17. O'Neil PM, Rieder S. Utility and validity of the eating behavior inventory in clinical obesity research: a review of the literature. Obes Rev 2005 Aug;6(3):209-216. [doi: 10.1111/j.1467-789X.2005.00192.x] [Medline: 16045636]

18. O'Neil PM, Currey HS, Hirsch AA, Malcolm RJ, Sexauer JD, Riddle FE, et al. Development and validation of the eating behavior inventory. Journal of Behavioral Assessment 1979 Jun;1(2):123-132. [doi: 10.1007/BF01322019]

\section{Abbreviations}

BMI: body mass index

PA: physical activity

UCSD: University of California, San Diego

WBI: Weight Behavior Inventory 
Edited by G Eysenbach; submitted 30.06.13; peer-reviewed by S Woolford, M Broom; comments to author 28.08.13; revised version received 05.09.13; accepted 17.09.13; published 06.11.13

Please cite as:

Kolodziejczyk JK, Norman GJ, Barrera-Ng A, Dillon L, Marshall S, Arredondo E, Rock CL, Raab F, Griswold WG, Sullivan M, Patrick $K$

Feasibility and Effectiveness of an Automated Bilingual Text Message Intervention for Weight Loss: Pilot Study

JMIR Res Protoc 2013;2(2):e48

URL: http://www.researchprotocols.org/2013/2/e48/

doi: 10.2196/resprot.2789

PMID: 24200517

CJulia K Kolodziejczyk, Gregory J Norman, Angelica Barrera-Ng, Lindsay Dillon, Simon Marshall, Elva Arredondo, Cheryl L Rock, Fred Raab, William G Griswold, Mark Sullivan, Kevin Patrick. Originally published in JMIR Research Protocols (http://www.researchprotocols.org), 06.11.2013. This is an open-access article distributed under the terms of the Creative Commons Attribution License (http://creativecommons.org/licenses/by/2.0/), which permits unrestricted use, distribution, and reproduction in any medium, provided the original work, first published in JMIR Research Protocols, is properly cited. The complete bibliographic information, a link to the original publication on http://www.researchprotocols.org, as well as this copyright and license information must be included. 